\title{
ANDES

www.scielo.cl

\section{Muerte súbita inesperada en la infancia: actualización y medidas de prevención}

\author{
Sudden unexpected death in infancy: update and preventive measures
}

\author{
Javier Cepeda S., ${ }^{\mathrm{a}, \mathrm{b}}$, Daniel Zenteno A., ${ }^{\mathrm{a}, \mathrm{b}}$, Claudia Fuentes S., ${ }^{\mathrm{a}, \mathrm{b}}$, Pablo Brockmann V.c
}

\begin{abstract}
aUnidad de Ventilación Mecánica Prolongada, Servicio de Pediatría, Hospital Clínico Regional Dr. Guillermo Grant Benavente. Concepción, Chile bepartamento de Pediatría, Facultad de Medicina, Universidad de Concepción. Concepción, Chile

‘Departamento de Cardiología y Respiratorio Pediátrico, Centro del Sueño, Escuela de Medicina, Pontificia Universidad Católica de Chile. Santiago, Chile
\end{abstract}

Recibido: 5 de octubre de 2020; Aceptado: 16 de marzo de 2021

\section{¿Qué se sabe del tema que trata este estudio?}

La muerte súbita inesperada de la infancia es una de las principales causas de muerte en menores de 1 año a nivel global, cuya incidencia se ha reducido drásticamente tras introducción de la campaña "Back to Sleep", promoviendo el sueño en supino.

\section{¿Qué aporta este estudio a lo ya conocido?}

Este estudio aporta un resumen actualizado de la evidencia reciente disponible relacionada con la muerte súbita inesperada en la infancia, con foco en la nueva terminología en uso, hallazgos fisiopatológicos y factores de riesgo, así como medidas de protección.

\section{Resumen}

La muerte súbita e inesperada en la infancia (MSII) definida como la muerte de un menor de 1 año durante el sueño sin motivo evidente de manera inicial, sigue siendo una de las causas más frecuentes de mortalidad infantil tardía. Aproximadamente 3.500 lactantes mueren anualmente en Estados Unidos por muertes relacionadas con el sueño. Se postula un origen complejo y multifactorial en un lactante vulnerable, sin embargo, su fisiopatología no se ha logrado comprender por completo. Los profesionales de la salud juegan un rol clave en la promoción de las medidas preventivas descritas en la literatura que incluyen dormir en posición supina sobre una superficie firme, evitar el tabaquismo y el colecho, promover la lactancia materna, entre otros. El objetivo de esta revisión es resumir las principales características epidemiológicas y fisiopatológicas de la MSII y factores relacionados con el sueño seguro.
Palabras clave: Síndrome de Muerte Súbita Infantil; Posición Prona; Epidemiología; Sueño Seguro; Lactante 


\section{Abstract}

Sudden unexpected death in infancy (SUDI) - defined as the death of a child under 1 year of age during sleep with no initially obvious cause - remains one of the most common causes of post-neonatal mortality. Approximately 3,500 infants die annually in the United States from sleep-related deaths. A complex and multifactorial origin is postulated in a vulnerable infant. However, the pathophysiology of SUDI has not been fully understood. Health care providers play a key role in promoting preventive measures described in the literature, which include sleeping in a supine position on a firm surface, avoiding smoking and co-sleeping, promoting breastfeeding, among others. The objective of this review is to summarize the main epidemiological and physiopathological characteristics of SUDI, and safe sleep-related factors.

\section{Keywords:}

Sudden Infant Death;

Prone Position;

Epidemiology;

Safe Sleep; Childhood

\section{Introducción}

El término síndrome de muerte súbita del lactante (SMSL) hace referencia a la muerte súbita e inesperada de un lactante menor de 1 año, que ocurre aparentemente durante el sueño y persiste sin explicación tras una exhaustiva investigación que incluye la historia clínica, una autopsia completa y la revisión de las circunstancias de la muerte ${ }^{1}$. Este síndrome fue definido inicialmente en el año 1969 y desde entonces ha sido modificado por diversos consensos de expertos a nivel internacional ${ }^{2}$.

Recientemente, el término muerte súbita inesperada en la infancia (MSII) o SUDI (sudden unexpected death in infancy) ha reemplazado al de SMSL. Este concepto hace referencia más bien a aquellas circunstancias en las que la causa de la muerte no es evidente en forma inicial, y por tanto incluye el SMSL (Clasificación Internacional de Enfermedades, décima revisióncódigo R95), pero también a otras categorías como las infecciones o anomalías anatómicas y del desarrollo no conocidas antes del fallecimiento (código R99), accidentes por prácticas de sueño no seguro y lesiones no accidentales descubiertas con posterioridad (código W75) $)^{1,3}$.

En noviembre del 2018 se llevó a cabo el Tercer Congreso Internacional de Muerte Súbita en niños e infantes en el instituto Radcliffe de la Universidad de Harvard. Su realización estuvo motivada por el creciente rechazo al diagnóstico de SMSL entre la comunidad médica, llevando a una falsa disminución en su reporte, debilitando su validez y confiabilidad. Las principales recomendaciones propuestas para la nueva versión del CIE-11, a implementarse el año 2022, fueron el cambio de término "SMSL" por "Muerte Súbita Inexplicable de la infancia" en la categoría MH11, evitando el uso de acrónimos que pueden dificultar su interpretación (ejemplificando con la letra " $U$ " en el acrónimo SUDI que puede generar confusión con los términos "unexpected", "undetermined", "unknow" o "unascertained"). Así mismo, se definieron los tér- minos "inesperado", "explicable", "indeterminado" e "inexplicable" de uso común, revisando, clasificando y recomendando terminología relativa al proceso de determinación de los casos 4 .

El objetivo del presente documento es realizar una revisión bibliográfica actualizada de las recientes definiciones de asociados al concepto de MSII con foco en su epidemiología, hallazgos fisiopatológicos, así como los factores de riesgo y protectores.

\section{Metodología}

Se realizó una revisión bibliográfica en PUBMED, LILACS y EMBASE combinando los términos libres y MESH: "Sudden infant death syndrome", "Bed sharing", "Prone position", "Epidemiology", "Sudden unexpected death in infancy". El periodo de búsqueda fue entre enero del 2000 y septiembre del 2020. Se incluyeron artículos publicados en inglés y español, limitados a menores de 1 año. En base a esto se redujo un total de 329 artículos a 43, excluyendo además aquellos en que no fue posible acceder al artículo completo o no existió relación entre el artículo y la revisión. Adicionalmente, se amplió la búsqueda a las referencias de los trabajos seleccionados. Los artículos seleccionados correspondieron a revisiones bibliográficas, estudios clínicos y revisiones sistemáticas, los que fueron analizados por 4 autores. La decisión final de su inclusión en la presente revisión narrativa estuvo basada en la impresión subjetiva de los autores.

\section{Epidemiología}

Desde una perspectiva histórica, la creciente evidencia de que la posición durante el sueño estaba relacionada al SMSL llevó a la Academia Americana de Pediatría (AAP) en 1992 a desaconsejar la posición prona durante el sueño y posteriormente a la introducción de la campaña "Back to Sleep" en 1994 (actual "Safe to 
Sleep") que buscaba concientizar respecto a los principales factores de riesgo de SMSL. Dicha intervención marcó una importante reducción en la incidencia de SMSL en Estados Unidos, pasando de 130 muertes por cada 100.000 nacidos vivos ( \pm 5.000 ) en 1990 a 35,4 muertes por cada 100.000 nacidos vivos el 2017 ( \pm $1400)^{5,6}$. En Europa, entre los años 2005-2015, la MSII correspondió al 9,7\% del total de muertes en el menor de 1 año, con una incidencia de 34,9 por cada 100.000 recién nacidos vivos, siendo la segunda causa más frecuente de mortalidad infantil tardía (defunción entre los 28 días - 11 meses de vida) en los países de Europa del Este, correspondiendo a la principal causa en Bélgica, Finlandia, Francia y Reino Unido ${ }^{7}$.

En Chile, Brockmann y cols., informaron un total de 1.442 casos de SMSL entre 1997-2009, 81\% de ellos en menores de 2 meses (menor edad que lo descrito en la experiencia internacional), con una razón varones a niñas de 1,42:1. La tasa de mortalidad nacional por SMSL fue de 0,45 por cada 1000 nacidos vivos, siendo mayor en las regiones del sur de Chile ${ }^{8}$.

A nivel mundial las tasas de MSII se han mantenido prácticamente invariables desde el 2006 a la fecha. Por otra parte, en consideración a una mayor consistencia en el estudio de la escena de muerte, ha existido un cambio diagnóstico con un incremento en la incidencia de las muertes atribuidas a sofocación accidental y estrangulación en la cama, así como por patología bien definida, manteniendo las tasas de MSII estables?.

La incidencia de SMSL se concentra en los primeros 6 meses de vida, con un máximo entre los 2 a 4 meses, afectando a familias de todos los grupos sociales, económicos y étnicos. Se describe una mayor incidencia en hijos de madre con inadecuado control prenatal, madres fumadoras durante el embarazo, recién nacidos de sexo masculino (relación hombre:mujer de $3: 2$ ), posición prono y lateral durante el sueño, recién nacidos de pretérmino o bajo peso al nacer y algunas etnias como los nativos americanos y lactantes blancos no - hispanos ${ }^{10}$.

Actualmente el SMSL es la cuarta causa de muerte en lactantes en Estados Unidos y la primera en aquellos entre 1 mes y 1 año de vida, con más de 1300 muertes por año $(0,35 \text { muertes por cada } 1000 \text { nacidos vivos })^{11}$.

El mayor reconocimiento de muertes en lactantes relacionadas a asfixia y estrangulamiento durante el sueño motivó a la AAP a actualizar sus recomendaciones y enfocarse en un ambiente de sueño seguro y no sólo en el SMSL ${ }^{12}$.

\section{Fisiopatología}

Considerando que el SMSL es un diagnóstico de exclusión, ha existido una intensa investigación en búsqueda de los mecanismos que puedan fundamentar los factores de riesgo conocidos, siendo más probable un origen multifactorial. La hipótesis del triple riesgo, el modelo más ampliamente aceptado, propone que cuando un lactante vulnerable, como un recién nacido de pretérmino o expuesto al tabaco materno, se encuentra en un periodo crítico e inestable del desarrollo del control homeostático ( 2 a 4 meses) y es expuesto a un estresor exógeno, como el ser posicionado en prono durante el sueño, es posible desencadenar el SMSL. El modelo propone que el SMSL pudiera conducir a la muerte sólo si estos 3 factores están presentes. La vía final del SMSL está ampliamente relacionada con una inmadurez del control cardiorrespiratorio en conjunto con una falla del mecanismo del despertar ${ }^{13}$. El apoyo a esta hipótesis proviene de numerosos estudios fisiológicos que sugieren la posibilidad de una fragilidad orgánica en la vía del despertar, particularmente en la sustancia gris periacueductal del mesencéfalo, el que se relaciona con una respuesta autonómica de activación o "respuesta visceral de alerta". Esta respuesta autonómica ocurre dentro de un sistema aferente de acetilcolina y el núcleo tegmental pedúnculo-pontino (NTP). El NTP provee acetilcolina al mesencéfalo, y su deficiencia podría inducir un desbalance en el sistema colina/monoamina en las víctimas de SMSL ${ }^{14}$.

Por otra parte, los factores extrínsecos proveen un gatillo ambiental al lactante vulnerable. La posición prona sigue siendo el factor de riesgo más importante de SMSL mediante el incremento de la posibilidad de re-inhalación de gases espirados, sofocación, sobrecalentamiento y alteración del mecanismo del desper$\operatorname{tar}^{1,2,3,5}$. Los principales mecanismos fisiopatológicos propuestos se detallan en la tabla 1.

\section{Factores de riesgo y protectores}

El conocimiento respecto de los factores de riesgo y protectores proviene principalmente de estudios de casos y controles, los que pueden determinar asociaciones, pero no son capaces de establecer causalidad. Los principales factores relacionados con el SMSL se resumen en la tabla 2 .

\section{Posición al dormir}

Las primeras asociaciones entre SMSL y posición prona fueron identificadas en 1965 y corroboradas posteriormente por múltiples estudios de casos y controles en Europa, Australia y Nueva Zelanda. En la década de los 80 , múltiples estudios demostraron que el dormir en posición prona aumentaba el riesgo de SMSL en aproximadamente 10 veces $^{1,3}$.

La posición prona se encuentra asociada a un incremento del riesgo de hipercapnia con la subsecuente hipoxia, disminución de la oxigenación cerebral, incremento de la temperatura corporal, alteración del 
Tabla 1. Fisiopatología del Síndrome de Muerte Súbita del Lactante ${ }^{1,2,3,5}$

\begin{tabular}{|c|c|c|}
\hline Mecanismo & Hallazgos & Fisiopatología propuesta \\
\hline \multirow[t]{4}{*}{ Asfixia } & Edema pulmonar en fallecidos por SMSL & \multirow{2}{*}{$\begin{array}{l}\text { Reinhalación de dióxido de carbono, con hipercapnia } \\
\text { e hipoxia }\end{array}$} \\
\hline & \multirow{3}{*}{$\begin{array}{l}\text { Niveles elevados de factor vascular endotelial, lactato } \\
\text { y eritropoyetina } 2^{\circ} \text { a hipoxia crónica }\end{array}$} & \\
\hline & & $\begin{array}{l}\text { Disminución del surfactante secundarios a hipoxia } \\
\text { crónica y menor compliance pulmonar }\end{array}$ \\
\hline & & Menor capacidad pulmonar $2^{\circ}$ a hipoxia in útero \\
\hline $\begin{array}{l}\text { Falla del mecanismo } \\
\text { de despertar }\end{array}$ & Elevación del umbral de despertar vs sueño en prono & $\begin{array}{l}\text { Falla del mecanismo que gatilla el despertar en lactan- } \\
\text { tes estresados que duermen en posición prono }\end{array}$ \\
\hline \multirow{4}{*}{$\begin{array}{l}\text { Alteración de la regulación } \\
\text { autonómica }\end{array}$} & \multirow{2}{*}{$\begin{array}{l}\text { Metabolitos de serotonina elevados, polimorfismos de } \\
\text { proteína transmembrana y menor densidad de seroto- } \\
\text { nina en sitios de unión a receptor }\end{array}$} & Menor efecto excitatorio en bulbo raquídeo \\
\hline & & $\begin{array}{l}\text { Menor serotonina extracelular por polimorfismos con } \\
\text { actividad promotora }\end{array}$ \\
\hline & \multirow[t]{2}{*}{ Disminución del neuropéptido orexina } & $\begin{array}{l}\text { Alteración de la termostasis y desrregulación del ritmo } \\
\text { cardiaco }\end{array}$ \\
\hline & & Elevación del umbral del despertar \\
\hline \multirow[t]{3}{*}{ Inmadurez neuronal } & Disminución del antígeno nuclear neuronal & \multirow[t]{3}{*}{ Desconocida } \\
\hline & Alteración de la mielinización & \\
\hline & Incremento en la apoptosis celular en tronco cerebral & \\
\hline Arritmias & Alteración del SCN5A & QTc prolongado por canalopatía (canal de sodio) \\
\hline \multirow[t]{2}{*}{ Infecciones } & Incremento del interferón gamma e IL-6 & \multirow{2}{*}{$\begin{array}{l}\text { Alteración de la respuesta a citoquinas e inmunomo- } \\
\text { dulación del Sistema Nervioso Central }\end{array}$} \\
\hline & $\begin{array}{l}\text { Mayor colonización bacteriana por St. aureus en po- } \\
\text { sición prono }\end{array}$ & \\
\hline
\end{tabular}

SMSL: Síndrome de muerte súbita del lactante

\section{Tabla 2. Factores de riesgo y protectores relacionados con el SMSL}

\begin{tabular}{|c|c|}
\hline Factores & $\begin{array}{c}\text { Odds ratio ( } 95 \% \text { Intervalo } \\
\text { de confianza) }\end{array}$ \\
\hline \multicolumn{2}{|l|}{ Factores de riesgo } \\
\hline Nacimiento antes de las 37 semanas $^{2}$ & $11,67(1,84-74,14)$ \\
\hline Colecho en el menor de 12 semanas $^{23}$ & $10,37(4,44-24,21)$ \\
\hline Colecho con madre fumadora ${ }^{23}$ & $6,27(3,94-9,99)$ \\
\hline Dormir sobre superficies blandas 25 & $5,1(3,1-8,3)$ \\
\hline Colecho ${ }^{23}$ & $2,89(1,99-4,18)$ \\
\hline Posición prona al dormir ${ }^{17}$ & $2,6(1,5-4,5)$ \\
\hline Exposición a tabaco 29 & $2,44 \%$ IC $95 \%(2,31-2,57)$ \\
\hline Dormir en decúbito lateral ${ }^{17}$ & $2,0(1,2-3,4)$ \\
\hline Raza negra9 ${ }^{9}$ & $1,96(N D)$ \\
\hline Sexo masculino ${ }^{11}$ & 1,7 (ND) \\
\hline Escaso nivel socioeconómico² & $1,24(1,15-1,34)$ \\
\hline \multicolumn{2}{|l|}{ Factores protectores } \\
\hline \multicolumn{2}{|c|}{ Lactancia materna exclusiva a los 2 meses $^{32} 0,6(0,44-0,82)$} \\
\hline Uso de chupete 35 & $0,5(N D)$ \\
\hline
\end{tabular}

ND: No disponible. SMSL: Síndrome de muerte súbita del lactante. control autonómico del sistema cardiovascular e incremento del umbral del despertar ${ }^{15,16}$. Se ha demostrado que el riesgo del sueño en prono (OR: 2,6 95\% IC 1,5$4,5)$ y en posición lateral (OR: 2,0 95\% IC 1,2-3,4) para el desarrollo de muerte súbita son similares, lo que se explica por la inestabilidad de la posición lateral que implica una alta probabilidad de girar hacia la posición prono $^{17}$. Se ha descrito una mayor probabilidad de ubicar al lactante en posición en prono o decúbito lateral en cuidadores nuevos, así como en las guarderías donde tienden de cambiar de posición a lactantes habituados al sueño en supino ${ }^{18}$.

Algunos padres han mostrado preocupación por el posible riesgo de asfixia cuando el lactante duerme en posición supina. No obstante, la anatomía de la vía aérea y su relación con el esófago hace menos probable la asfixia en esta posición. La justificación yace en que al ubicarse en posición supina, la vía aérea se encontrará por sobre el esófago permitiendo que cualquier regurgitación sea rápidamente deglutida, evitando así la aspiración ${ }^{1,19}$.

En Chile, un estudio piloto realizado en 100 lactantes menores de 45 días evidenció que un 80\% duerme en posición supina y un $20 \%$ en posición prono o la- 
teral, destacando una tasa de colecho de 30\%. Un 90\% de las familias había recibido información respecto de las medidas del sueño seguro, siendo este el principal predictor de dormir en posición supina ${ }^{20}$.

\section{Colecho}

El dormir en la misma superficie junto a otra persona se encuentra asociado a mayor riesgo de SMSL en estudios de casos y controles, pudiendo ser el factor de riesgo más importante en menores de 4 meses $^{21}$. Esto se explica por el uso de colchones blandos, el mayor riesgo de sobreabrigo y la posibilidad de rodar y cubrir al lactante durante el sueño. Por otra parte, desaconsejarlo puede ser controversial puesto que el colecho favorece la lactancia materna, la que es un conocido factor protector de $\mathrm{SMSL}^{22}$. En consideración a lo anterior, compartir la habitación, es decir, dormir en proximidad al lactante permitiendo una adecuada observación, siendo capaz de escucharlo y tocarlo, sin compartir la misma cama, parece ser la medida más adecuada ${ }^{1}$. Adicionalmente, el riesgo de morir al compartir la misma cama es mayor si uno de los padres es fumador, cuando la madre ha fumado durante el embarazo, el recién nacido es prematuro o con bajo peso al nacimiento, si el adulto consume alcohol o drogas que alteran los mecanismos del despertar, se duerme sobre un sofá, la cama es blanda, el colecho es durante toda la noche y el lactante es menor de 11 semanas $^{23,24}$.

\section{Ropa de cama}

$\mathrm{Al}$ evaluar el escenario de muerte, hasta un $25 \%$ de los lactantes víctimas de SMSL son encontrados con sus cabezas cubiertas por ropas de cama ${ }^{25}$. El uso de ropas de cama de consistencia blanda (frazadas, almohadas, pieles de oveja) y posicionadores en el ambiente de sueño, ha sido asociado con un incremento de hasta 5 veces del riesgo de SMSL independiente de la posición al dormir y más de 20 veces si el lactante duerme en prono. Adicionalmente, predisponen a un mayor riesgo de sobreabrigo, reinhalación y son capaces de cubrir la cabeza durante el sueño ${ }^{26}$.

Estudios han evidenciado que el uso de ropas de cama de consistencia blanda es el factor de riesgo más importante en mayores de 4 meses, pues estas tienen la capacidad de girar hacia estas superficies, pero son incapaces de liberarse de ellas ${ }^{21}$.

Si bien es más seguro que un lactante duerma sin frazadas, si se utilizan, estas deben ser delgadas y los lactantes deben ser posicionados con sus pies tocando la parte más baja de la cuna, haciendo pasar el cubrecama bajo las axilas. Si bien esta práctica parece lógica, siendo observada en casos de lactantes no afectados por SMSL en estudios de casos y controles, aún no existen estudios que la avalen como una estrategia protectora ${ }^{2}$.

Recientemente, el uso sacos para dormir ha sido recomendado por algunas organizaciones relacionadas con SMSL. Un saco de dormir se podría considerar seguro si no permite al lactante deslizarse dentro de éste, ni que su cabeza sea cubierta. El saco debe ser del tamaño correcto para cada lactante, con cuello ajustable y mangas para mantener el calor, pero sin capucha. No obstante, existe escasa evidencia que demuestre un efecto protector ${ }^{27}$.

La práctica de envolver, "fajar" o "enlular" al lactante es utilizada con el fin de ayudarlo a conciliar el sueño. Si se realiza, debe hacerse colocando al lactante en posición supina y debe de suspenderse de manera inmediata ante cualquier indicio de que el lactante es capaz de girar. Un meta-análisis reciente evaluó que el mayor riesgo de SMSL es al utilizarlo en posición prono (OR = 12,99 IC 95\% 4,14 - 40,77), seguido de la posición de lado $(\mathrm{OR}=3,16 \mathrm{IC} 95 \%$ 2,08 - 4,81) y posición supina $(\mathrm{OR}=1,93 \mathrm{IC} 95 \%$ 1,27 - 2,93). Existe evidencia que sugiere que el riesgo de SMSL con esta práctica aumenta con la edad, duplicándose en mayores de 6 meses $^{28}$.

\section{Tabaquismo materno}

Numerosos estudios han demostrado que el humo del cigarro es capaz de incrementar el riesgo de SMSL en hasta 5 veces. En un estudio reciente, Anderson y cols, analizaron la base de datos de mortalidad infantil del Center for Disease Control and Prevention (CDC) entre el 2007 y 2011 demostrando un incremento lineal del riesgo de MSII con el consumo de tabaco en el embarazo, duplicándose al consumir 1 cigarro diario (OR ajustado 1,98, IC 95\% 1,73 - 2,28) y aumentando en 0,07 por cada cigarro por sobre $1^{29}$. Adicionalmente a la reducción de la distensibilidad y volúmenes pulmonares, la exposición in útero posee un efecto neurotóxico, lo que lleva a una alteración del mecanismo del despertar y disminución la variabilidad cardiaca en respuesta al estrés lo que altera la capacidad de respuesta al medio ambiente ${ }^{30}$.

Si bien es difícil separar las consecuencias de la exposición ambiental al tabaco en lactantes de la exposición prenatal, se describe también su influencia sobre la función pulmonar y un incremento del riesgo de SMSL en un efecto dosis dependiente ${ }^{3}$.

Un estudio chileno estimó que el consumo prenatal de tabaco estuvo relacionado a un 40\% del total de casos de SMSL ${ }^{31}$. Por tanto, los padres y especialmente la madre, deben ser motivados al cese absoluto del hábito tabáquico durante el embarazo y después del parto y no permitir que nadie fume en el ambiente que circunda al lactante.

\section{Prematurez}

La prematurez y el bajo peso al nacimiento incrementan en 4 veces el riesgo de sufrir SMSL en com- 
paración a los recién nacidos de término y el riesgo es inversamente proporcional a la edad gestacional. Gran parte de este riesgo se deriva de un sistema autonómico inmaduro, que conduce a una alteración de los mecanismos del despertar y un incremento de la hipercapnia $^{32}$. Pese a que la prematurez se encuentra asociada a apneas, no existe evidencia de que estas apneas precedan al SMSL, motivo por el cual los monitores no se recomiendan de manera rutinaria en la prevención del SMSL $^{12}$.

Los prematuros se encuentran en igual o mayor riesgo de ser posicionados en prono, probablemente como continuación de lo observado por los padres en las unidades de neonatología. Se recomienda que los prematuros sean posicionados en supino tan pronto como se encuentre clínicamente estables, preferiblemente a partir de las 32 semanas de edad post-menstrual, a fin de habituar a los padres previo al alta hospitalaria $^{33}$.

\section{Lactancia materna}

La lactancia materna tiene un efecto protector contra el SMSL. Un meta-análisis reciente con evaluación de los datos individuales de 8 estudios de casos y controles, con un total de 2.267 casos, demostró que la lactancia materna de $\geq 2$ meses de duración fue protectora. Se observó una relación directamente proporcional entre duración de la lactancia materna y el grado de protección de $\mathrm{SMSL}^{34}$.

\section{Inmunizaciones}

Estudios de casos y controles y análisis de reportes de efectos adversos a vacunas en Estados Unidos no han demostrado un efecto positivo de las inmunizaciones en la prevención del SMSL ${ }^{3}$. No obstante, un meta-análisis encontró que el riesgo de SMSL disminuye en hasta en un 50\% al seguir adecuadamente un plan de vacunación, especialmente las vacuna contra la difteria, tétanos y tos ferina (DPT) y polio oral ${ }^{35}$. El aumento en la cobertura de DPT se encuentra inversamente relacionado con mortalidad por MSII, con una tasa de reducción en la incidencia de 0,92 por cada 100.000 por cada $10 \%$ de incremento en la cobertura de la población ${ }^{36}$. Por tanto, el miedo subsecuente de desarrollar SMSL no puede ser nunca un argumento en contra de la inmunización regular.

\section{Uso de chupete}

Si bien su mecanismo protector es poco claro, se cree que su uso favorece los mecanismos del despertar, incrementa la presión arterial durante el sueño y mejora el control autonómico de la frecuencia cardiaca. Una revisión reciente de la literatura analizó 59 publicaciones, incluyendo 11 trabajos observacionales y 3 meta-análisis, demostrando una reducción de hasta el 50\% del riesgo de SMSL sin interferir en el establecimiento de la lactancia materna ${ }^{37}$. No obstante, una revisión Cochrane del año 2017 no encontró ningún estudio randomizado seleccionable para establecer recomendaciones $^{38}$. La AAP recomienda su uso toda vez que la lactancia materna este completamente establecida y en el momento en el que el lactante sea posicionado para dormir sin necesidad de reinsertarlo si este cae de la boca, ni forzarlo si lo rechaza ${ }^{12}$.

La recomendación de expertos de la AAP publicada el 2016, actualiza las recomendaciones con el objetivo de reducir las muertes relacionadas con el sueño en lactantes, incluyendo el SMSL. Estas se basan en estudios de casos y controles en menores de 1 año y la fuerza de la recomendación está basado en el sistema SORT (Strength of Recomendation Taxonomy). El resumen de las recomendaciones se describe en la tabla $3^{12}$.

SMSL, eventos de aparente amenaza de la vida (ALTE) y eventos breves, resueltos e inexplicados (BRUE)

A excepción de los factores previamente mencionados, no existe forma de predecir que lactante fallecerá por $\mathrm{SMSL}^{39}$. El ALTE/BRUE no ha demostrado a la fecha estar relacionado con el SMSL, siendo el tabaquismo materno el único factor de riesgo común a ambas condiciones ${ }^{40}$. Estudios en búsqueda de alguna relación entre ALTE y SMSL han determinado que solo un $4-13 \%$ de los pacientes con SMSL tiene historia de apnea, ligeramente superior al de los controles ${ }^{40,41}$. En la actualidad no se recomienda el uso rutinario de monitores, pues no han demostrado ser beneficiosos en la prevención del SMSL ${ }^{12}$.

\section{Colapso subito e inesperado postnatal, MSII y piel con piel}

El colapso súbito e inesperado postnatal (CSIP) incluye tantos los casos de ALTE severo como los de MSII que ocurren durante la primera semana de vida y corresponde aproximadamente al 5\% del total. Por su parte, el "piel con piel" hace referencia a la práctica de posicionar al recién nacido desnudo en prono sobre el torso de la madre inmediatamente o poco después del nacimiento ${ }^{42}$.

Múltiples series de casos de CSPI han sido reportados de recién nacidos aparentemente sanos durante la práctica de piel con piel en posición prona, principalmente durante las 2 primeras horas de vida, relacionados con sofocación o atrapamiento. La AAP sugiere que la forma de realizar un piel con piel seguro es 
Tabla 3. Resumen de recomendaciones de la AAP para la prevención del SMSL ${ }^{37}$

\begin{tabular}{lc}
\hline Recomendación & Grado de evidencia SORT \\
\hline Dormir de espalda & $\mathrm{A}$ \\
Utilizar una superficie firme al dormir & $\mathrm{A}$ \\
Lactancia materna & $\mathrm{A}$ \\
Cohabitar y dormir en superficies separadas & $\mathrm{A}$ \\
Alejar superficies blandas & $\mathrm{A}$ \\
Chupete al dormir & $\mathrm{A}$ \\
Evitar la exposición al tabaco ante y postnatal & $\mathrm{A}$ \\
Evitar el consumo de alcohol y drogas & $\mathrm{A}$ \\
Evitar el sobreabrigo & $\mathrm{A}$ \\
Adecuado control prenatal & $\mathrm{A}$ \\
Inmunización completa según calendario & $\mathrm{A}$ \\
Educación por el personal de atención neonatal & $\mathrm{A}$ \\
"Tiempo boca abajo" en vigilia & $\mathrm{B}$ \\
Evitar dispositivos comerciales inconsistentes con las recomendaciones de sueño seguro & $\mathrm{B}$ \\
Fajar o "enlular" & $\mathrm{C}$ \\
\hline
\end{tabular}

SORT: Taxonomía de la fuerza de recomendación. SMSL: Síndrome de muerte súbita del lactante.

mantenerse el contacto visual entre recién nacido y la madre, ubicarlo en posición de olfateo, con hombros y torso enfrentados a la madre, evitar cubrir la nariz y boca, girar la cabeza hacia un costado, evitar la flexión cervical, manteniendo un monitoreo continuo por parte del personal de salud en la sala de parto y de manera regular en postparto, permitiendo que la madre duerma, para lo que se deberá ubicar al recién nacido en su cuna y bajo la supervisión de otra persona alerta y despierta ${ }^{43}$.

\section{Conclusiones}

La MSII es un trastorno complejo y multifactorial, que requiere un continuo estudio para establecer sus bases fisiopatológicas e interacciones que conducen a la vulnerabilidad de los lactantes, periodos críticos del desarrollo y factores de riesgo ambiental. A la fecha, variables epidemiológicas junto con diversas intervenciones basadas en la evidencia, han permitido reducir su incidencia. Si bien es improbable eliminar comple- tamente el riesgo de MSII, se espera con una adecuada educación e implementación de las medidas de sueño seguro, partiendo desde el hospital hasta el domicilio, este riego se pueda reducir al máximo.

Es importante que el personal de salud entregue un mensaje consistente y sin contradicciones, basado en las recomendaciones publicadas. Particularmente importantes son las recomendaciones relacionadas con el sueño en posición supina sobre una superficie firme, evitar la exposición al tabaco, favorecer la lactancia materna y evitar el colecho, reconociendo además aquellas sin evidencia, como el uso de monitores.

Finalmente, las futuras investigaciones deberán enfocarse en cómo las variables fisiopatológicas alteran la respuesta fisiológica típica, así como en establecer las mejores y más efectivas formas de llevar a cabo campañas educacionales a la población.

\section{Conflicto de intereses}

Los autores declaran no tener conflicto de intereses.

\section{Referencias}

1. Horne R. Sudden infant death syndrome: current perspectives. Intern Med J. 2019;49(4):433-8.

2. Adams S, Ward C, Garcia K. Sudden infant death syndrome. Am Fam Physician. 2015;91(11):778-83.

3. Carlin R, Moon R. Risk Factors, Protective Factors, and Current
Recommendations to Reduce Sudden Infant Death Syndrome: A Review. JAMA Pediatr. 2017;171(2):175-80.

4. Goldstein R, Blair P, Sens M, et al. Inconsistent classification of unexplained sudden deaths in infants and children hinders surveillance, prevention and research: recommendations from The 3rd International Congress on Sudden Infant and Child Death. Forensic Sci Med
Pathol. 2019;15(4):622-8.

5. Goldberg N, Rodriguez-Prado Y, Tillery R, et al. Sudden Infant Death Syndrome: A Review. Pediatr Ann. 2018;47(3):e118-e123.

6. Center for Disease Control and Prevention. About sudden unexpected infant death and sudden infant death syndrome. https://www.cdc.gov/sids/data. htm. Consultado en Julio, 2020. 
7. de Visme S, Chalumeau M, Levieux $\mathrm{K}$, et al. National Variations in Recent Trends of Sudden Unexpected Infant Death Rate in Western Europe. J Pediatr. 2020;226:179-85

8. Brockmann P, Oyarzún M, Villarroel $\mathrm{L}$, et al. Síndrome de muerte súbita del lactante: prevalencia y cambios en los últimos años en Chile. Rev Med Chile. 2013;141(5):589-94.

9. Matthews T, MacDorman M, Thoma M. Infant Mortality Statistics From the 2013 Period Linked Birth/Infant Death Data Set. Natl Vital Stat Rep. 2015;64(9):1-30.

10. Heron M. Deaths: Leading Causes for 2014. Natl Vital Stat Rep. 2016;65(5):1-96.

11. US Center for Disease Control and Prevention. Infant deaths: linked birth/ infant death records. https://wonder.cdc. gov/lbd.html. Consultado en Julio, 2020.

12. Moon R; task force on sudden infant death syndrome. SIDS and Other SleepRelated Infant Deaths: Evidence Base for 2016 Updated Recommendations for a Safe Infant Sleeping Environment. Pediatrics. 2016;138(5):e20162940.

13. Filiano J, Kinney H. A perspective on neuropathologic findings in victims of the sudden infant death syndrome: the triplerisk model. Biol Neonate. 1994;65(34):194-7.

14. Sawaguchi T, Franco P, Kato I, et al. From physiology to pathology: arousal deficiency theory in sudden infant death syndrome (SIDS)--with reference to apoptosis and neuronal plasticity. Forensic Sci Int. 2002;130 Suppl:S37-S43.

15. Kanetake J, Aoki Y, Funayama M. Evaluation of rebreathing potential on bedding for infant use. Pediatr Int. 2003;45(3):284-9.

16. Wong F, Witcombe N, Yiallourou S, et al. Cerebral oxygenation is depressed during sleep in healthy term infants when they sleep prone. Pediatrics. 2011;127(3):e558-e565.

17. Li D, Petitti D, Willinger $\mathrm{M}$, et al. Infant sleeping position and the risk of sudden infant death syndrome in California, 1997-2000. Am J Epidemiol. 2003;157(5):446-55.

18. Moon R, Patel K, Shaefer S. Sudden infant death syndrome in child care settings. Pediatrics. 2000;106(2 Pt 1):295-300.

19. Jeffery H, Megevand A, Page H. Why the prone position is a risk factor for sudden infant death syndrome. Pediatrics. 1999;104:263-9.

20. Sanchez T, Peirano D, Pipino C, et al. Bad Sleeping habits in infants: risk factors for sudden infant death syndrome. Rev Chil Pediatr. 2020;91(4):529-35.

21. Colvin J, Collie-Akers V, Schunn $\mathrm{C}$, et al. Sleep environment risks for younger and older infants. Pediatrics. 2014;134(2):e406-e412.

22. Hauck F, Thompson JM, Tanabe K, et al. Breastfeeding and reduced risk of sudden infant death syndrome: a meta-analysis. Pediatrics. 2011;128(1):103-10.

23. Carpenter R, Irgens L, Blair P, et al. Sudden unexplained infant death in 20 regions in Europe: case control study. Lancet. 2004;363(9404):185-91.

24. Vennemann M, Hense H, Bajanowski T, et al. Bed sharing and the risk of sudden infant death syndrome: can we resolve the debate? J Pediatr. 2012;160(1):44-8.e2.

25. Blair P, Mitchell E, Heckstall-Smith EM, et al. Head covering - a major modifiable risk factor for sudden infant death syndrome: a systematic review. Arch Dis Child. 2008;93(9):778-83.

26. Hauck F, Herman S, Donovan M, et al. Sleep environment and the risk of sudden infant death syndrome in an urban population: the Chicago Infant Mortality Study. Pediatrics. 2003;111(5 Pt 2):120714.

27. Finlay F. Can infant sleeping bags be recommended by medical professionals as protection against sudden infant death syndrome? Arch Dis Child. 2019;104(3):305-7.

28. Pease A, Fleming P, Hauck F, et al. Swaddling and the risk of sudden infant death syndrome: a meta - analysis. Pediatrics 2016; 137:e20153275.

29. Anderson T, Lavista Ferres J, Ren S, et al. Maternal Smoking Before and During Pregnancy and the Risk of Sudden Unexpected Infant Death. Pediatrics. 2019;143(4):e20183325.

30. Richardson H, Walker A, Horne R Maternal smoking impairs arousal patterns in sleeping infants. Sleep. 2009;32(4):515-21.

31. Cerda J, Bambs C, Vera C. Infant morbidity and mortality attributable to prenatal smoking in Chile. Rev Panam Salud Publica. 2017;41:e106.

32. Ostfeld B, Schwartz-Soicher O, Reichman
$\mathrm{N}$, et al. Prematurity and sudden unexpected infant deaths in the United States. Pediatrics 2017;140: e20163334.

33. American Academy of Pediatrics Committee on Fetus and Newborn. Hospital discharge of the high-risk neonate. Pediatrics. 2008;122(5):1119-26.

34. Thompson J, Tanabe K, Moon R, et al. Duration of Breastfeeding and Risk of SIDS: An Individual Participant Data Meta-analysis. Pediatrics. 2017;140(5):e20171324.

35. Vennemann M, Höffgen M, Bajanowski $\mathrm{T}$, et al. Do immunisations reduce the risk for SIDS? A meta-analysis. Vaccine. 2007;25(26):4875-9.

36. Muller-Nordhorn J, Hettler-Chen C, Keil $\mathrm{T}$, et al. Association between suddent infant death syndrome and diphtheriatetanus-pertussis inmunisation: an ecology study. BMC Pediatr. 2015;15(1):1.

37. Alm B, Wennergren G, Möllborg P, et al. Breastfeeding and dummy use have a protective effect on sudden infant death syndrome. Acta Paediatr. 2016;105(1):318

38. Psaila K, Foster JP, Pulbrook N, et al. Infant pacifiers for reduction in risk of sudden infant death syndrome. Cochrane Database Syst Rev. 2017;4(4):CD011147.

39. Tieder J, Altman RL, Bonkowsky J, et al. Management of apparent life-threatening events in infants: a systematic review. J Pediatr. 2013;163(1):94-9.e96.

40. Behnam-Terneus M, Clemente M. SIDS, BRUE, and Safe Sleep Guidelines. Pediatr Rev. 2019;40(9):443-55.

41. Zenteno Araos D, Díaz Silva J, Brockmann Veloso P. Aplicación de una nueva terminología "BRUE: eventos breves, resueltos e inexplicados". Definiciones y recomendaciones. Rev Chil Pediatr. 2020;91(3):424-31.

42. Herlenius E, Kuhn P. Sudden unexpected postnatal collapse of newborn infants: a review of cases, definitions, risks, and preventive measures. Transl Stroke Res. 2013;4(2):236-47.

43. Feldman-Winter L, Goldsmith J; Committee on Fetus And Newborn; Task Force on Sudden Infant Death Syndrome. Safe Sleep and Skin-toSkin Care in the Neonatal Period for Healthy Term Newborns. Pediatrics. 2016;138(3):e20161889. 